\title{
A NOTE ON MINIMAL PRIME IDEALS
}

\author{
D. D. ANDERSON
}

(Communicated by Eric Friedlander)

\begin{abstract}
Let $R$ be a commutative ring and $I$ an ideal of $R$. We show that if all the prime ideals minimal over $I$ are finitely generated, then there are only finitely many prime ideals minimal over $I$.
\end{abstract}

Let $R$ be a commutative ring with identity, and let $I \neq R$ be an ideal of $R$. It is well known that if $R$ is Noetherian then there are only finitely many prime ideals minimal over $I$. In fact, we only need $R$ to satisfy the ascending chain condition on radical ideals [1, Theorem 88]. Of course, for $R$ Noetherian every prime ideal minimal over $I$ is finitely generated. The purpose of this note is to show that for any commutative ring if every prime ideal minimal over $I$ is finitely generated, then there are only finitely many prime ideals minimal over $I$. It is interesting to note that if we take $R$ to be the ring of all sequences from $Z / 2 Z$ that are eventually constant, with pointwise addition and multiplication, then $R$ is a zero-dimensional Boolean ring with minimal prime ideals $M_{i}=\left\{\left\{a_{n}\right\} \in R \mid a_{i}=0\right\}$ and $M_{\infty}=\left\{\left\{a_{n}\right\} \in R \mid a_{n}=0\right.$ for large $n\}$ and each $M_{i}$ is principal but $M_{\infty}$ is not finitely generated. Thus while $R$ has infinitely many minimal primes ideals, only one is not finitely generated.

Theorem. Let $R$ be a commutative ring with identity, and let $I \neq R$ be an ideal of $R$. If every prime ideal minimal over $I$ is finitely generated, then there are only finitely many prime ideals minimal over $I$.

Proof. Let $S=\left\{P_{1} \cdots P_{n} \mid\right.$ each $P_{i}$ is a prime ideal minimal over $\left.I\right\}$. If for some $C=P_{1} \cdots P_{n} \in S$ we have $C \subseteq I$, then any prime ideal $P$ minimal over $I$ contains some $P_{i}$, so $\left\{P_{1}, \ldots, P_{n}\right\}$ is the set of minimal prime ideals of $I$. Hence we may assume that $C \notin I$ for each $C \in S$. Consider the set $T=\{J \mid J$ is an ideal of $R$ with $J \supseteq I$ and $C \notin J$ for each $C \in S\}$ partially ordered by $\subseteq$. Since each element of $S$ is finitely generated, $T$ is inductive and hence by Zorn's Lemma has a maximal element $Q$. It is easily seen that $Q$ is prime. But then since $Q \supseteq I, Q$ contains a prime ideal $P$ minimal over $I$ [1, Theorem 10]. Thus $P \in S$, a contradiction.

Received by the editors November 18, 1992.

1991 Mathematics Subject Classification. Primary 13A15. 


\section{REFERENCES}

1. I. Kaplansky, Commutative rings, revised edition, Univ. of Chicago Press, Chicago, IL, 1974.

Department of Mathematics, University of Iowa, Iowa City, Iowa 52242

E-mail address: ddanders@umaxc . weeg . uiowa.edu 